35 Abg. Schewe-Gerigk, BT-Plenarprotokoll 16/70 vom 30. November 2006, S. 6955.

36 Abg. Wunderlich, BT-Plenarprotokoll 16/70, S. 6953.

37 Abg. Lambrecht (SPD), BT-Plenarprotokoll 16/70, S. 6956.

38 Freudenberg, NJ 2006, 535, 538.

39 BT-Drs. $16 / 575$, S. 8.

40 Mitsch, NJW 2007, 1237, 1241.

41 Mitsch, NJW 2007, 1237, 1241 sowie ferner noch Mosbacher, NStZ 2007, 665,670 , der insofern zwar $\$ 238$ Abs. 4 StGB für sachgerecht hält, zugleich aber die Einstufung als Privatklagedelikt wegen des damit für das Opfer verbundenen Kostenrisikos für nicht ganz glücklich hält und anzweifelt.

42 Vgl. zu den folgenden Zitaten jeweils Buettner, ZRP 2008, 124 ff. Zust. Rackow, GA 2008, 552, 568 in Fn. 125.

43 BT-Drs. 16/3663, S. 2.

44 Buettner, ZRP 2008, 124 (Hervorhebung nicht im Original).

45 Vgl. die Zahlen bei $V o ß$, Stellungnahme zur öffentlichen Anhörung im Rechtsausschuss vom 18. Oktober 2006 zum Thema „Stalking“ unter 3. (Beziehungskonstellationen Opfer-Täter).

46 S. zur Einteilung von Stalker-Typen und deren Motiven näher Müller in: Krüger, Stalking als Straftatbestand, S. 25 ff. m. w. N. insbesondere zum soziologischen Schrifttum. Dass diese Einteilung im Rahmen der vorliegenden (Teil-)Problematik durchaus von Interesse ist, wurde an gleicher Stelle dargetan, vgl. Krüger, Stalking als Straftatbestand, S. 198.

47 Buettner, ZRP 2008, 124, 125 (Hervorhebung nicht im Original).

48 Obwohl Nr. 86 RiStBV bloß das „öffentliche Interesse“ im Sinne von \$ 376 StPO näher erläutert, können deren Begriffserläuterungen - trotz der unterschiedlichen Begrifflichkeit - durchaus auf das „besondere öffentliche Interesse“ gemäß $\$ 238$ Abs. 4 StGB übertragen werden. Im Bereich der einfachen und fahrlässigen Körperverletzungsdelikte geht man jedenfalls von einer weitgehenden Identität beider Begriffe aus, wie die Lektüre der Nr. 233 ff. RiStBV zeigt.

49 Mitsch, NJW 2007, 1237, 1241 sowie ferner noch Mosbacher, NStZ 2007, 665, 670 und im selben Sinne bereits Fünfsinn, NK 2005, 82, 83.

50 In dieser Richtung, wenngleich es sich dabei um ein generelles Problem bei Antragsdelikten handelt, bereits Janovsky, Stellungnahme zur öffentlichen Anhörung im Rechtsausschuss vom 18. Oktober 2006 zum Thema „Stalking“, S. 4: „Beeinflussungen durch den Täter“ sowie Hecht, Berliner Interventionszentrale bei häuslicher Gewalt, Stellungnahme zur öffentlichen Anhörung im Rechtsausschuss vom 18. Oktober 2006 zum Thema „Stalking“, S. 12: „Einflussnahme des Täters auf das Opfer“.

51 Esser/Wolmerath, Mobbing, 2. Aufl., S. 292; Bieszk/Sadtler, NJW 2007, 3382, 3383.

52 Vgl. dazu Mühe, Mobbing am Arbeitsplatz - Strafbarkeitsrisiko oder Strafbarkeitslücke, 2006, S. 55 f.; Bieszk/Sadtler, NJW 2007, 3382, 3386 f. sowie - auf Basis des $\$ 238$ Abs. 1 Nr. 5 StGB - ferner noch Krüger, Stalking als Straftatbestand, S. $148 \mathrm{f}$.

53 Hoffmann, Stalking, S. 162.

54 Gass in: Dreßing/Gass (Hrsg.), Stalking, S. 73.
55 S. hierzu bloß Krüger, Stalking als Straftatbestand, S. 164 ff. m. w. N.

56 BVerfGE 80, 367, 379; BGHSt 19, 325, 332 ff.; 34, 397, 401; Beulke, Strafprozessrecht, 10. Aufl., $\mathbb{2} 23$ Rdnr. 473; Hellmann, Strafprozessrecht,

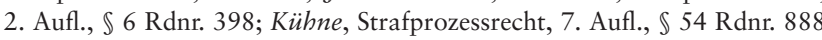
- jeweils m. w. N.

57 BVerfGE 80, 367, 381

58 Nach Geppert, Grundsatz der Unmittelbarkeit im Strafverfahren, 1979, S. 181 (Hervorhebung im Original) soll zwar jener zentrale $\$ 250 \mathrm{StPO}$ nicht schlechthin Sitz des Unmittelbarkeitsprinzips sein, sondern bloß einen Teilbereich von (materieller) Unmittelbarkeit regeln. Gleichwohl lässt sich aber nicht ernsthaft bestreiten, dass die Norm eine gesetzliche Ausprägung dieser Verfahrensmaxime ist.

59 BGHSt 6, 141, 143; 20, 160, 161; BGH NStZ 1982, 79; Meyer-Goßner, \250 Rdnr. 8; Gollwitzer in: LR, 25. Aufl., \ 251 Rdnr. 7; Schneidewin, JR 1951, 481, 483; Wömpner, NStZ 1983, 293, 294. Wenn man mit der Gegenansicht eine Unterscheidung zwischen zu Beweiszwecken angefertigten und anderweitig hergestellten „schriftlichen Erklärungen“ von vornherein nicht zulassen will (s. dazu etwa Krause, Urkundenbeweis im Strafprozeß, 1966, S. 154 ff., 159), kommt $\$ 250$ Satz 2 StPO erst Recht zum Zuge.

60 Meyer-Goßner, $\mathbb{} 250$ Rdnr. 8; Schneidewin, JR 1951, 481, 483.

61 BGH GA 1962, 339; Stree in: Schönke/Schröder, $\$ 46$ Rdnr. 41; Franke in: MünchKommStGB, $\mathbb{S} 46$ Rdnr. 48, wohingegen der umgekehrte Schluss nicht zulässig ist, vgl. dazu bloß BGH, a. a. O. sowie StV 1987, 100.

62 Bestimmte Ausnahmen vom Unmittelbarkeitsprinzip werden jedenfalls mit Opferschutz begründet, wie es insbesondere für die Videovernehmung und deren gesetzliche Lozierung in $\$ 255$ a StPO zutrifft, vgl. dazu etwa Mildenberger, Schutz kindlicher Zeugen im Strafverfahren durch audiovisuelle Medien, 1995, S. 230 ff.; Nowak, Der gefährdete Zeuge im deutschen und polnischen Strafverfahren, 2000, S. 72 ff.; Swoboda, Videotechnik im Strafverfahren, 2002, S. 399 ff. Gerade die Videovernehmung des StalkingOpfers könnte sich unter Opferschutzgesichtspunkten anbieten. Wenn man sich dabei noch vor Augen führt, dass der Bundesrat in seinem - sogleich noch darzustellenden - Gesetzentwurf zur Einführung eines Rechtsbeistands für Opfer von schwerem Stalking diesen in einem Atemzug mit den

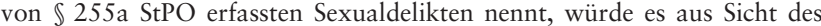
Gesetzgebers durchaus Sinn machen, wenn er zu einer entsprechenden Novellierung dieser Vorschrift schreiten würde.

63 Gass in: Dreßing/Gass (Hrsg.), Stalking, S. 73. Vgl. ferner Hoffmann, Stalking, S. 162: Dokumentation des Geschehens als eine oft nicht unbeträchtliche Belastung für die Opfer.

64 BT-Drs. 16/9448.

65 Meyer-Goßner, $\mathbb{S} 397$ a Rdnr. 3 a. E., wie es bei Stalking-Opfern aus denselben Gründen, warum das (besondere) öffentliche Interesse anzunehmen ist, durchaus nahe liegt.

66 BT-Drs. 16/9448, S. 7.

67 Ebda., S. 6 (Hervorhebung nicht im Original).

68 Vgl. zum Folgenden jeweils BT-Drs. 16/9448, S. 8.

\title{
Nachträgliche Sicherungsverwahrung - eine empirische erste Bilanz
}

Michael Alex

\section{Ausgangslage}

Am 29.07.2004 wurde mit dem $\$ 66b StGB die nachträgliche Sicherungsverwahrung im deutschen Strafrecht eingeführt, nachdem bereits seit 2001 einzelne Bundesländer (Baden-Württemberg, Bayern, Sachsen-Anhalt, Niedersachsen, Thüringen) polizeirechtliche Regelungen zur Unterbringung im Strafvollzug nach vollständiger Strafvollstreckung getroffen hatten. Diese Ländergesetze hatte das Bundesverfassungsgericht am 10.02.2004 wegen Verstoßes gegen Art. 74 GG für verfassungswidrig erklärt und dem Bundesgesetzgeber empfohlen, bis 30.09.2004 eine strafrechtliche Lösung zu finden. Die Regelung in $\ 66 \mathrm{~b}$ StGB geht infolge ihrer Dauerwirkung deutlich weiter als die von den Nationalsozialisten in Art. 5 des „Gewohnheitsverbrechergesetzes“ vom 24.11.1933 (RGBl 1933, 995) getroffene Übergangslösung für Verurteilungen aus der Weimarer Republik.

Vier Jahre nach Inkrafttreten des $\$ 66 \mathrm{~b}$ StGB kann eine erste Bilanz gezogen werden. Nach den vorliegenden Unterlagen und veröffentlichten Entscheidungen sind von 2001 (zunächst auf Grundlage von Straftäter-Unterbringungsgesetzen einzelner Bundesländer) bis Ende 2006 gut 110 Anträge auf Anordnung der nachträglichen Unterbringung durch die Gerichte zurückgewiesen worden, darunter knapp 40, die auf Grundlage der Straftäter-Unterbringungsgesetze 
in Bayern und Baden-Württemberg gestellt worden waren ${ }^{1}$. Infolge der restriktiven Auslegung des Begriffs „nach der Verurteilung erkennbare Tatsachen“, die sog. Nova, durch die Rechtsprechung ist die nachträgliche Sicherungsverwahrung bisher erst in 7 Fällen vom Bundesgerichtshof rechtskräftig bestätigt worden (Antwort der Bundesregierung vom 22.05.2008 auf eine kleine Anfrage der Fraktion DIE LINKE, BT-Drucks. 16/9241), wobei in einem dieser Fälle das Bundesverfassungsgericht die Entscheidung des BGH aufhob (Beschluss vom 23.08.2006, NStZ 2007, 87 ff.). Damit hat sich die von Frommel (2004) gehegte Hoffnung, die Gerichte würden den $\ 66 \mathrm{~b}$ StGB auf den rechtsstaatlich vertretbaren Kern reduzieren, erfüllt.

Dennoch hat sich der Alltag im Justizvollzug nachhaltig verändert, und die Bindung von Ressourcen bei Justiz und Sachverständigen ist erheblich. So müssen in einigen Bundesländern die Justizvollzugsanstalten routinemäßig bei der Staatsanwaltschaft einen Antrag auf Anordnung der nachträglichen Sicherungsverwahrung anregen, sobald die formalen Voraussetzungen des $\ 66$ b StGB erfüllt sind, und die Staatsanwaltschaft tritt in eine Vorprüfung ein, über deren Ergebnis sie wiederum dem Justizministerium berichten muss (vgl. Stellungnahme Kreuzer anlässlich der Anhörung im Rechtsausschuss des Bundestages am 28.05.2008). Angesichts der geringen praktischen Bedeutung der Vorschrift infolge der restriktiven Rechtsprechung scheint ein solcher Aufwand völlig unverhältnismäßig, abgesehen von den Belastungen für Gefangene und Personal in den Justizvollzugsanstalten. Insofern fällt die Bilanz für die populistische Gesetzgebungsmaschinerie der vergangenen 10 Jahre verheerend aus. Das gilt umso mehr, als $\mathbb{6} 66 \mathrm{~b}$ StGB auch im Hinblick auf das angebliche Ziel, die "Sicherheitslage“ zu verbessern, unnötig und ungeeignet zu sein scheint, wie von den meisten Experten erwartet worden ist. Das legen die Ergebnisse einer Totalerhebung des Lehrstuhls für Kriminologie der Ruhr-Universität Bochum nahe, die zum Ziel hatte, alle Fälle zu untersuchen, in denen Anträge auf Anordnung der nachträglichen Sicherungsverwahrung letztendlich durch die Rechtsprechung zurückgewiesen worden sind mit der Folge, dass die Gefangenen bis zum 31.12.2006 aus dem Strafvollzug entlassen worden sind.

\section{Untersuchung zur Rückfallhäufigkeit nach abgelehnter Unterbringung}

Von den Justizministerien der Bundesländer bzw. den zuständigen Staatsanwaltschaften wurden auf Anfrage Daten über 89 entsprechende Fälle übermittelt. Aus unterschiedlichen Gründen konnten allerdings aus Baden-Württemberg keine Daten zu verweigerten Unterbringungen nach dem dortigen StrUBG erlangt werden, und auch aus Bayern wurden in 16 von 21 Fällen nach dem BayStrUBG keine weitergehenden Auskünfte erteilt. Damit verbleiben 67 Fälle, bei denen ein Abgleich mit aktuellen Auszügen aus dem Bundeszentralregister möglich war, in 62 Fällen wurde darüber hinaus Einsicht in Vollstreckungshefte bzw. Verfahrensakten gewährt. Im Hinblick auf die Fragestellung waren 6 Vollstreckungshefte nur begrenzt ergiebig, weil die Staatsanwaltschaft in diesen Fällen auf Anregung der JVA nur eine Vorprüfung vorgenommen, aber keinen Antrag auf Anordnung der nachträglichen Sicherungsverwahrung gestellt hat. Insgesamt dürften die von Gerichtsentscheidungen nach $\$ 66 \mathrm{~b}$ StGB betroffenen Haftentlassenen fast vollständig von der Untersuchung erfasst worden sein, lediglich bei den abgelehnten Unterbringungen nach den Straftäter-Unterbringungsgesetzen in Baden-Württemberg und Bayern gibt es größere Lücken. Das ist für eine Langzeitstudie aber weniger bedeutsam, weil nach den Ländergesetzen enger als in \66b StGB die Gefahr „gegenwärtig“ sein musste, der Rückfall also unmittelbar nach der Entlassung zu erwarten war.

Der Abgleich mit dem Bundeszentralregister ergab in den Fällen, in denen ausreichende Auskünfte für eine Anfrage beim Bundesamt für Justiz erteilt worden waren, folgendes Bild zur Rückfallhäufigkeit der Entlassenen:

Tab. 1: Strafhöhe bei erneuter Verurteilung $(n=67)$

\begin{tabular}{|c|c|c|c|}
\hline \multicolumn{2}{|l|}{ Erneute Verurteilung zu } & Keine erneute Eintragung bis 30.06.2008 \\
\hline Geldstrafe & Freiheitsstrafe m. Bewährung & Freiheitsstrafe o. Bewährung & \\
\hline 9 & 4 & 10, davon 2 zusätzlich SV & 44 \\
\hline
\end{tabular}

Tab. 2: Delikte bei Verurteilung zu Freiheitsstrafe ohne Bewährung und Strafmaß

\begin{tabular}{|l|l|l|}
\hline Delikt & Strafmaß & Einschätzung der Gefährlichkeit durch Gutachter \\
\hline Diebstahl & 4 Monate & hoch \\
\hline Diebstahl, BtMG & 7 Monate und 2 Wochen & Kein Gutachten erstellt \\
\hline Diebstahl, BtMG & 10 Monate & Kein Gutachten erstellt \\
\hline Betrug, BtMG, Weisungsverstoß geg. FA & 1 Jahr und 4 Monate & mittel \\
\hline $\begin{array}{l}\text { Gemeinschaftlicher Raub, } \\
\text { gef. Körperverletzung }\end{array}$ & 2 Jahre und 2 Monate & hoch \\
\hline Gemeinschaftl. Diebstahl & 2 Jahre und 6 Monate & hoch \\
\hline Gewerbsm. Handel BtM & 3 Jahre und 6 Monate & hoch \\
\hline $\begin{array}{l}\text { Betrug, Einbruchsdiebst., Urkunden- } \\
\text { fälschung }\end{array}$ & 4 Jahre & hoch/mittel \\
\hline $\begin{array}{l}\text { Schw. sex. Missbrauch von Kindern } \\
\text { Schwere räub. Erpressung }\end{array}$ & $\begin{array}{l}\text { 7 Jahre und } \\
\text { Bicherungsverwahrung }\end{array}$ & hicherungsverwahrung \\
\hline
\end{tabular}


Einzelheiten hinsichtlich der Verurteilungen zu Freiheitsstrafen ohne Bewährung ( $\mathrm{n}=10)$ sind in der folgenden Übersicht dargestellt:

Lediglich drei Delikte sind Katalogtaten des $\$ 66 \mathrm{~b}$ StGB, erreichen also die dort geforderte Erheblichkeit für die Integrität der Opfer. Einschränkend ist allerdings darauf hinzuweisen, dass erst gut $1 / 4$ der Probanden $(\mathrm{n}=18)$ vor mehr als 3 Jahren aus der Haft entlassen worden sind und 5 der 10 Verurteilungen zu unbedingten Freiheitsstrafen auf diese kleine Gruppe entfallen (deshalb soll der Abgleich mit dem Bundeszentralregister in drei Jahren wiederholt werden). In den kommenden Jahren sind also noch einige weitere Rückfälle zu erwarten, in Einzelfällen lassen sich den Unterlagen bereits jetzt neue Haftbefehle und (nicht rechtskräftige) Urteile entnehmen.

Einige Tendenzen sind aber schon jetzt erkennbar. So ist bemerkenswert, dass bisher kaum bedeutsame Verurteilungen registriert sind und dass 44 Haftentlassene, bei denen zumindest die Justizvollzugsanstalten, in der Regel aber auch die Staatsanwaltschaft eine hohe Gefährlichkeit für erhebliche Delikte angenommen hatte, in den 5 - $1 \frac{1}{1 / 2}$ Jahren seit ihrer Entlassung strafrechtlich überhaupt nicht wieder registriert worden sind. Ebenso bedeutsam ist, dass bei 28 der bisher nicht wieder registrierten 44 Entlassenen von zumindest einem der beauftragten Sachverständigen eine hohe Gefährlichkeit prognostiziert worden war (in 10 dieser Fälle wurden keine Gutachten erstellt oder die Unterlagen enthielten dazu keine Angaben).

Anlassdelikte für den Antrag auf Anordnung der nachträglichen Sicherungsverwahrung waren in erster Linie sexueller Missbrauch ( $\mathrm{n}=$ 22), Vergewaltigung $(\mathrm{n}=17)$, Tötungsdelikte $(\mathrm{n}=12)$ und Raub ( $\mathrm{n}=$ 12). Unter Berücksichtigung der von Jehle et al. (2003) vorgelegten Zahlen zur Rückfälligkeit bei diesen Delikten binnen vier Jahren mit einem Anteil von $15-26 \%$ zu erneuten Freiheitsstrafen Verurteilten liegt die Stichprobe der mit nachträglicher Sicherungsverwahrung bedrohten Haftentlassenen bisher eher an der unteren Grenze. Ihr Risiko, erneut erhebliche Straftaten zu begehen, liegt also nicht höher als bei allen anderen Haftentlassenen. Daran wird deutlich, dass es offenbar keine trennscharfen Kriterien für „eine erhebliche Gefährlichkeit“ im Sinne des $\$ 66$ b StGB gibt.

Vor diesem Hintergrund - immer unter dem Vorbehalt, dass Katamnesezeiträume unter 5 Jahren gerade bei Sexual- und Tötungsdelikten als zu kurz angesehen werden (vgl. Dahle 2004) - zwingt der hohe Anteil bisher „falsch Positiver“ auch zu einer besonders kritischen Betrachtung der Sachverständigengutachten. Häufigste Diagnose war die „dissoziale Persönlichkeitsstörung “ $(\mathrm{n}=32)$, und 15 der erneut Verurteilten, also 2/3, sind mit dieser Diagnose belegt worden (5 Geldstrafen, 4 bedingte Freiheitsstrafen, 5 unbedingte Freiheitsstrafen, 1 Freiheitsstrafe zuzüglich Sicherungsverwahrung). Andererseits sind in dieser Kategorie auch beinahe $40 \%$ der Haftentlassenen erfasst, die bisher nicht erneut strafrechtlich in Erscheinung getreten sind. Mindestens ebenso sicher könnte man sich einfach auf die justizielle Vorbelastung der erneut Verurteilten stützen. 17 der 23 neuen Verurteilungen gehen auf das Konto von Haftentlassenen, die vor dem Indexdelikt mindestens fünfmal vorbestraft waren $(n=41)$. So sehr die Standards der Gutachtenerstellung in den vergangenen Jahren auch verbessert worden sein mögen (vgl. Boetticher et al. 2006, Bock 2007), machen diese Zahlen bereits deutlich, wie groß die Gefahr ist, gerade mit so „schwammigen“ Störungsbildern wie der „dissozialen Persönlichkeitsstörung“ Menschen fälschlich eine hohe Gefährlichkeit zu attestieren.

Das wird bestätigt durch die Ergebnisse einer Studie mit einer relativ kleinen Stichprobe $(\mathrm{n}=33)$ von in der DDR abgeurteilten Rechts- brechern, die nach der Wiedervereinigung zunächst gem. $\$ 63$ in den Maßregelvollzug gekommen und auf Grundlage von zwei Entscheidungen des Bundesverfassungsgerichts Mitte der 90er Jahre wegen formeller Verfahrensfehler zu entlassen waren (Rusche 2004). Nur 8 von diesen von mehreren Gutachtern als gefährlich eingeschätzten Entlassenen wurden binnen 6 Jahren überhaupt rückfällig $(24,2 \%)$, 5 von ihnen begingen gefährliche Straftaten überwiegend auf dem Gebiet der Gewalt- und Sexualdelinquenz, die erneute Unterbringung oder empfindliche Haftstrafen zur Folge hatten. Damit unterschieden sie sich kaum von einer gleich großen Kontrollgruppe, die regulär entlassen worden waren. Man kann deshalb davon ausgehen, dass ebenso wie in anderen Ländern in Deutschland $85 \%$ der vermeintlich gefährlichen Gefangenen/Patienten unnötig untergebracht sind (Rusche 2004). Standardisierte Prognoseverfahren wie HCR-20 oder PCL sind zwar geeignet, „Gefährliche“ zu erkennen (alle Rückfälligen in jener Untersuchung hatten einen hohen score), aber nur auf Kosten der „Ungefährlichen“, die ebenfalls hohe Werte erzielten, ohne dass sich das in einem Rückfall manifestierte. Die Zahl der auf dieser Grundlage falsch positiv eingeschätzten Patienten (15) war sogar größer als bei den internen Klinikprognosen mit herkömmlicher klinischer Diagnostik (11).

\section{Fazit}

Besorgniserregend ist vor dem Hintergrund der durchgeführten Untersuchung, dass die Zahl der in der Sicherungsverwahrung Untergebrachten von 182 im Jahre 1990 auf 427 am 31.03.2007 angestiegen ist und bei der Stichtagserhebung des Statistischen Bundesamtes am 31.03.2008 sich bereits 435 Untergebrachte im Justizvollzug befanden. Man muss also davon ausgehen, dass derzeit in den Abteilungen für Sicherungsverwahrte ca. 360 Menschen untergebracht sind, die keine Gefahr für die Gesellschaft darstellen. Gleichzeitig macht dieses Missverhältnis deutlich, dass trotz aller Bemühungen um standardisierte Verfahren den Sachverständigen noch immer keine Instrumente zur Verfügung stehen, die eine zuverlässige Prognose erlauben. Verantwortungsbewusste Sachverständige weisen immer wieder darauf hin, wie schwierig die Vorhersage menschlichen Verhaltens ist. Gleichwohl vermitteln die Gutachten häufig den Eindruck, es beständen keinerlei Zweifel an der Richtigkeit der Prognose. Durch die strengen Anforderungen der Rechtsprechung an die formalen Voraussetzungen einer dauerhaften Unterbringung haben wir erstmalig in größerem Umfang in Deutschland Verhältnisse, wie sie aus dem Baxtrom-Experiment im Staat New York bekannt sind: eine Vielzahl vermeintlich gefährlicher Inhaftierter muss aufgrund der Rechtslage entlassen werden, so dass nachprüfbar wird, wie zutreffend die Prognose gewesen ist. Die Zwischenergebnisse der hier vorgestellten Untersuchung zur nachträglichen Sicherungsverwahrung legen die Vermutung nahe, dass eine erhöhte Gefährlichkeit bei dem Personenkreis, der für die nachträgliche Sicherungsverwahrung in Betracht kommt, nicht gegeben ist. Dafür spricht auch, dass bei 39 der 67 Entlassenen bereits bei der Anlassverurteilung die formalen Voraussetzungen für die Anordnung originärer Sicherungsverwahrung vorgelegen hatten, in einigen Fällen die Gerichte die Anordnung aber ausdrücklich abgelehnt hatten, weil offensichtlich seinerzeit der politische Druck noch wesentlich geringer war. Es ist deshalb an der Zeit, dass die Sachverständigen sich wieder in Bescheidenheit üben und die Begrenztheit ihrer Aussagen über das künftige Verhalten der Probanden ernsthaft betonen. Andernfalls machen sie sich zum Gehilfen für eine Kriminalpolitik, der es gleichgültig ist, wie viele Menschen unnötig auf Dauer vom gesellschaftlichen Le- 
ben ausgeschlossen werden (vgl. auch Pressemitteilung des Komitees für Grundrechte und Demokratie vom 03.07.2008, abrufbar unter: www.grundrechtekomitee.de). 25 der in dieser Studie Untersuchten waren bis zum Abschluss der Gerichtsverfahren über ihr reguläres Haftende hinaus untergebracht, vier von ihnen länger als 1 1/2 Jahre. Mit ein wenig Haftentschädigung ist es da nicht getan. Es ist Zeit für ein Umdenken! Die Folgerung des CDU-Politikers Jürgen Gehb (lt. Frankfurter Rundschau v. 24.07.2008) aus der restriktiven Rechtsprechung, die „neuen Erkenntnisse“ aus dem $\$ 66 \mathrm{~b}$ StGB zu streichen, ist sicher nicht der richtige Weg, sie wird der realen Bedrohung durch einschlägige Rückfälle auch nicht gerecht.

Michael Alex, Lehrstubl für Kriminologie, Kriminalpolitik, Polizeiwissenschaft, (Prof. Dr. Thomas Feltes M.A.) Ruhr-Universität Bochum, 44780 Bochum, Tel. 0234/32-28245/-25244, E-Mail: thomas.feltes@rub.de
Literaturangaben:

Bock 2007: Bock, Michael: Das Elend der klinischen Kriminalprognose, StV $5,2007,269$

Boetticher et al. 2006: Boetticher, Axel; Kröber, Hans-Ludwig; Müller-Isberner, Rüdiger; Böhm, Klaus M.; Müller-Metz, Reinhard; Wolf, Thomas: Mindestanforderungen für Prognosegutachten, NStZ 2006, 537.

Dahle 2006: Dahle, Klaus-Peter: Grundlagen und Methoden der Kriminalprognose. In: Kröber/Dölling/Leygraf/Sass (Hrsg.): Handbuch der Forensischen Psychiatrie, Band 3, Darmstadt 2006, 1 ff.

Frommel 2004: Frommel, Monika: Lebenslange Verwahrung angeblich nicht therapierbarer und extrem gefährlicher Sexualstraftäter seit 1998 in Deutschland und der Schweiz, NK 3/2004, 86.

Jehle et al. 2003: Jehle, Jörg-Martin; Heinz, Wolfgang; Sutterer, Peter: Legalbewährung nach strafrechtlichen Sanktionen - eine kommentierte Rückfallstatistik, Bonn, Mönchengladbach 2003.

Rusche 2004: Rusche, Stefan: In Freiheit gefährlich? - Eine Untersuchung zu Häufigkeit und Gründen falscher Kriminalprognosen bei psychisch kranken Gewaltverbrechern, Regensburg 2004.

\section{Fußnoten:}

1 Nach Berichten in „Der Spiegel“ v. 20.10.2003 und „Die Welt" vom 23.10.2003 waren in Bayern 30 Anträge zurückgewiesen worden, nach Auskunft der Bayerischen Staatsregierung vom 22.10.2007 lediglich 21.

\section{Sicherungsverwahrung gem. $\$ 7$ Abs. 2 JGG und der Präventionsgedanke im Strafrecht - kritische Betrachtung eines legislativen Kunstgriffs}

\section{Nina Nestler und Christian Wolf}

\section{Einleitung}

Mit dem Gesetz zur Einführung der nachträglichen Sicherungsverwahrung bei Verurteilungen nach Jugendstrafrecht vom 8.7.2008 ${ }^{1}$ fügte der Gesetzgeber einem umfassenden Projekt zur Reformierung des Straf- und Maßregelrechts ein weiteres Puzzleteil hinzu. Bereits seit Längerem konnte der aufmerksame Beobachter den Eindruck gewinnen, das Strafrecht mutiere mehr und mehr von einem Recht repressiver Sanktionen zu einer Art universell einsetzbarem, präventiv-sicherheitsrechtlichem „Breitschwert“ des Gesetzgebers. ${ }^{2}$ Eine besondere Rolle scheint dabei die Maßregel der Sicherungsverwahrung zu spielen, welche in den vergangenen zehn Jahren bereits ein bemerkenswertes Revival erlebte. Gemäß $\$ 7$ Abs. 2 JGG n. F. darf nun auch für nach Jugendstrafrecht Verurteilte nachträglich die Unterbringung in der Sicherungsverwahrung angeordnet werden, sofern nach der Verhängung einer Jugendstrafe von mindestens sieben Jahren wegen eines Verbrechens gegen das Leben, die körperliche Unversehrtheit, die sexuelle Selbstbestimmung oder nach $\$ \mathbb{S} 251$, 252, 255 StGB, durch welches das Opfer seelisch oder körperlich schwer geschädigt worden ist, vor Ende des Vollzugs Umstände erkennbar werden, die auf eine erhebliche Gefährlichkeit des Täters für die Allgemeinheit hinweisen.

Die Neuregelung des $₫ 7$ Abs. 2 JGG bildet ein Beispiel populistischer Ad-hoc-Aktionen des Gesetzgebers, mit dem er auf Beunruhigungen oder Ängste in der Bevölkerung reagiert. Unter dem Deckmantel eines vermeintlich gesteigerten Sicherheitsbedürfnisses ${ }^{3}$, das zu einem wesentlichen Teil jedoch aus überzogener medialer Berichterstattung resultiert ${ }^{4}$, antwortet man durch derlei Kunstgriffe auf scheinbare Bedrohungen um Handlungsstärke und Durchsetzungsfähigkeit zu demonstrieren.

II. Die Sicherungsverwahrung für Jugendliche im kriminalpolitischen Kontext

\section{Sicherungsverwahrung in Deutschland}

a) Die Entwicklung des Rechts der Sicherungsverwabrung für Erwachsene

Überlegungen zu einer dauerhaft sicheren Verwahrung nicht besserungsfähiger Gewohnheitsverbrecher zum Schutz der Allgemeinheit finden sich schon bei Franz von Liszt, der als mögliche Wirkungen der Strafe die Besserung, Abschreckung und Unschädlichmachung identifizierte. Gegen unverbesserliche Gewohnheitsverbrecher müsse sich die Gesellschaft schützen, schrieb von Liszt, wobei - da „köpfen und hängen “ sowie deportieren nicht in Betracht komme - eben „nur die Einsperrung auf Lebenszeit, bzw. auf unbestimmte Zeit“ bleibe. ${ }^{5}$ Mit dem Gesetz gegen gefährliche Gewohnheitsverbrecher und über Maßregeln der Besserung und Sicherung wurde in Deutschland das Institut der Sicherungsverwahrung in $\ 42 \mathrm{e}$ RStGB mit Wirkung zum 1.4.1934 eingeführt. ${ }^{6}$

Nach dem 2. Weltkrieg fand die Vorschrift des $\mathbb{4} 42 \mathrm{e}$ StGB ohne größere Änderungen in das bundesdeutsche Strafgesetzbuch Eingang. Die Justiz begegnete der Sicherungsverwahrung jedoch unter Verweis auf den vorangegangenen Missbrauch der Maßregel durch das NS-Regime mit größter Zurückhaltung. Diesen Bedenken und 